\title{
Diagnosis and management of tuberculous paraplegia with special reference to tuberculous radiculomyelitis
}

\author{
D A VID FREILICH A N MICHAEL SWASH \\ From the Department of Neurology, The London Hospital, London
}

SUMMARY Paraplegia occurred in eight of 17 patients with central nervous system tuberculosis. In six of these paraplegia was the presenting feature. Paraplegia may complicate tuberculous meningitis, or vertebral tuberculosis, but it may also occur, as in three of our cases, as a primary localised spinal tuberculous radiculomyelitis. These cases are presented in relation to the concept that paraplegia complicating these forms of tuberculosis is caused by radiculomyelitis.

Until recently tuberculous infection of the central nervous system appeared to be of decreasing importance in the United Kingdom, although it has remained a common clinical problem elsewhere, particularly in Asia (Dastur et al., 1968; Tandon and Pathak, 1973; Wadia, 1973a). In these countries tuberculous paraplegias caused by Pott's disease, extradural and intradural spinal tuberculomas, spinal radiculomyelitis, or other chronic forms of tuberculous meningitis are relatively common (Dastur and Wadia, 1969; Tandon and Pathak, 1973; Wadia, 1973b). Kocen and Parsons (1970) and McNicol et al. (1971) have described some features of the changing pattern of tuberculosis of the nervous system in Britain, particularly among non-European immigrants. In this paper we shall emphasise the importance of tuberculous radiculomyelitis (arachnoiditis), hitherto an almost unrecognised cause of paraplegia in Britain, and compare our recent experience with that at The London Hospital 50 years ago.

\section{Subjects}

The case records of all patients with tuberculous infection of the nervous system admitted to The London Hospital in the years 1972 to 1977 were reviewed. Seventeen patients with tuberculosis of the central nervous system were admitted in this six year period. Their ages ranged from 18 to 50 years (mean 46). Thirteen $(76 \%)$ were male.

Address for correspondence and reprint requests: $\mathrm{Dr}$ M. Swash, Department of Neurology, The London Hospital, Whitechapel, London E1 1 BB.

Aczepted 9 July 1978
Eleven (64\%) were immigrants; seven of the latter came from the Indian subcontinent. Two patients died. The clinical presentation of these 17 patients varied. Eight (47\%) presented with the syndromeo of tuberculous meningitis and six $(36 \%)$ witle $\vec{N}$ paraplegia caused by spinal tuberculosis. The re⿻ maining three patients had cerebral tuberculomas Eight patients (47\%) developed paraplegia.

\section{PARAPLEGIA COMPLICATING TUBERCULOUS} MENINGITIS

Two of the eight patients with tuberculous menin gitis developed a paraplegia during the course of the illness.

\section{Case 1}

This 34 year old Asian woman had migrated to the United Kingdom from India a year before admission to hospital. In August 1977 she was admitted to another hospital with tuberculous meningitis. For two weeks she had complained of anorexia, vomiting, headache, and remittent fever. On examination she was drowsy and confused, with marked stiffness, but there were no other signs. The CSF contained 160 lymphocytes $/ \mathrm{mm}^{3}$, $1.8 \mathrm{~g}$ protein/l, and $1.9 \mathrm{mmol}$ glucose $/ 1$. Acid-fast bacilli were not isolated from the CSF, but they were later cultured from the urine. The Mantoux test was postive at $1: 10000$. Treatment was begun with rifampicin, isoniazid, and pyridoxine. A week later bilateral lateral rectus palsies were noted. At this time the CSF protein was $3.6 \mathrm{~g} / 1$. She was treated with prednisolone (30 mg daily) for four weeks. She improved, and was discharged in October 1977, still taking antituberculous drugs.

In November 1977 she complained of pain in 
her back, radiating round her waist on both sides. Two weeks later she noted bilateral sciatic pain, and difficulty in walking. In the next few days her legs became weaker, and she was readmitted scarcely able to walk. Examination revealed a flaccid, areflexic paraparesis with slight distal sensory impairment to pinprick and touch in the legs below the knees. The right plantar response was extensor; the left was equivocal. The arms were normal, and there was no bladder disturbance. There was no spinal tenderness or deformity. The CSF contained 103 lymphocytes $/ \mathrm{mm}^{3}$ and $13.5 \mathrm{~g}$ protein/1 but the CSF glucose was normal and acid-fast bacilli were not isolated from the specimen. Plain radiographs of the spine and a myelogram were normal. Antituberculous chemotherapy was continued, and prednisone $(30 \mathrm{mg}$ daily) was begun. During the next month there was rapid improvement in strength, gait, and sensory disturbance. The tendon reflexes and the CSF abnormalities gradually resolved.

Comment Despite apparently successful management of her tuberculous meningitis this patient developed a flaccid, areflexic paraplegia, with only slight sensory disturbance, but with localised and radicular pain suggestive of a lesion at $\mathrm{T} 10$ root. These signs were preceded by an increase in the CSF protein. There was no radiographic evidence of vertebral tuberculosis, and the myelogram was normal. The clinical features were consistent with radiculomyelitis, and there was a rapid response to treatment with prednisone, while antituberculous drugs were continued.

\section{Case 2}

This 58 year old Asian man, who had entered Britain from Bangladesh 15 years previously, was transferred to The London Hospital for management of tuberculous meningitis. Eight months earlier he had complained of low back pain, but radiographs of the lumbar spine showed osteoarthrosis without other changes. For three months he had also complained of loss of appetite, and of increasingly severe right sided sciatica. It was thought that he had a lumbosacral disc prolapse but there was no improvement after a period of bed rest. He had been admitted to another hospital five weeks previously because of headache and intermittent fever. Examination was normal, but repeat radiographs of the lumbar spine showed erosion of the lower margin of the body of the second lumbar vertebra. The chest radiograph was normal. The CSF was clear and colourless, containing $0.4 \mathrm{~g}$ protein $/ 1$ and $2.2 \mathrm{mmol}$ glucose $/ 1$, but no cells. His fever continued. Two weeks later, neck stiffness was noted, and a second lumbar puncture showed yellow, viscous CSF containing 20 lymphocytes $/ \mathrm{mm}^{3}, 9.5 \mathrm{~g}$ protein $/ 1$, and 4.4 mmol glucose/1. Rifampicin, streptomycin, and isoniazid were begun, but he gradually deteriorated.

On admission to The London Hospital he was drowsy, mumbling incoherently. The right pupil was larger than the left, but both reacted to light. The fundi were normal. There was a flaccid areflexic paraparesis, more marked on the right. Both plantar responses were extensor. The bladder was palpable. Sensation to pinprick was impaired in both legs but no upper level could be defined. The arms were normal.

Prednisolone (20 mg daily) was started. A few days later left sided focal seizures developed. A lumbar puncture revealed yellow fluid under low pressure with spinal block. The CSF contained $16 \mathrm{~g}$ protein $/ 1,4.83 \mathrm{mmol}$ glucose $/ 1$, and 4 leucocytes/ $\mathrm{mm}^{3}$. Cerebral angiography showed narrowing of the right internal carotid artery at the base of the brain, irregular narrowing of the right middle cerebral artery, and slow perfusion of its branches. These appearances were considered consistent with arteritis.

The subsequent clinical course was complicated by hyponatraemia and seizures and, because of doubt about the diagnosis of tuberculous meningitis, a meningeal biopsy was performed through a right parietal burrhole. A granulomatous meningeal infiltrate was found in the biopsy but no acid-fast bacilli were seen. A ventricular reservoir was implanted and $10 \mathrm{mg}$ streptomycin was given into the ventricle daily for 10 days. He improved, but a year later he remained disabled by dementia and by weakness of the legs. The tendon reflexes had returned, the plantar responses were flexor, and sensory loss was no longer apparent. During this time the lesion of the second lumbar vertebra became calcified. Acid-fast bacilli were never isolated from CSF, sputum, or urine.

Comment In this patient the bony lesion, situated adjacent to the intervertebral disc, was characteristic of tuberculous infection and this was probably the site of invasion of the subarachnoid space, although acid-fast bacilli were never isolated. Sciatic pain early in the course of the illness was caused by nerve root involvement by tuberculoma or localised arachnoiditis, but the paraparesis which developed later resulted from cord involvement at a level much higher than that of the bony lesion. This was probably caused by vasculitis similar to that seen at angiography and in the cortical meningeal biopsy. The development of spinal block was an indication of impending paraplegia (Brooks et al., 1954). 
PARAPLEGIA COMPLICATING VERTEBRAL TUBERCULOSIS

The typical bony changes of vertebral tuberculosis may also be complicated by a paraplegia of mixed radicular and cord origin, but this is usually preceded by a phase of chronic spinal tuberculous meningitis.

Case 3

This 29 year old Asian nurse, born in St Lucia, had lived in England for eight years. In 1970 she had been treated for sarcoidosis, manifested by arthralgia, granulomatous uveitis, a persistent dry cough, pulmonary hilar lymphadenopathy, hypercalcaemia, and a raised ESR. During this illness the Mantoux test was negative, and the Kveim test positive. Her symptoms and signs gradually resolved with steroid therapy.

In February 1974 she noted persistent low back pain with left sided sciatica. There was no improvement after a period of bed rest and, in June 1974, a radiograph of the lumbar spine revealed collapse of the body of the fifth lumbar vertebra. The ESR was $75 \mathrm{~mm} / \mathrm{hr}$. During the next three weeks she developed weakness and numbness first of the left leg, and then of the right leg. A diagnosis of metastatic carcinoma was considered but no primary neoplasm could be found. The ESR rose to $108 \mathrm{~mm} / \mathrm{hr}$, and the Mantoux test became positive at $1: 10000$. A chest radiograph showed slight residual hilar lymphadenopathy. A lumbar puncture revealed turbid CSF containing 1000 leucocytes $/ \mathrm{mm}^{3}$ (mostly neutrophils), $5 \mathrm{~g}$ protein $/ 1$, and $2.67 \mathrm{mmol}$ glucose $/ 1$. Very scanty acid-fast bacilli were seen in smears of this specimen, and cultures were later reported positive.

The patient was transferred to The London Hospital. On examination at that time there was marked weakness of both legs, especially of hip flexion and of all movements at the ankles. She could not sit up unaided. Tone was reduced and both ankle jerks were absent. In the right leg there was sensory impairment in the L5, S1, and S2 dermatomes, and in the left leg in part of the L5 dermatome. The lower lumbar spine was tender and painful. Straight leg raising was limited by pain to about $20^{\circ}$. There was retention of urine, with incontinence. Treatment with rifampicin, ethambutol, and isoniazid was started and six weeks later her legs were stronger although distal weakness, especially of eversion and dorsiflexion at the ankles, remained prominent. The sensory disturbance improved, and bladder function returned. Some weeks later a spinal fusion was performed, and a sequestrum was removed from the body of the fifth lumbar vertebra. During the next few months there was an excellent functional recovery.

Comment In most reported cases of tuberculosis after sarcoidosis, both phases of the illness have affected the same organ, usually the lung (Scadding, 1967), but in our patient there was no clinical evidence of pulmonary tuberculosis. The subsequent vertebral tuberculous infection was only recognised when lumbar puncture showed the features of localised spinal tuberculous meningitis. The radiological feature of bony collapse was misleading, since tuberculosis of the spine more commonly presents with erosion of the margin of a vertebral body, as in our case 2 . Conservative management with antituberculous drugs, without myelography, followed by spinal fusion resulted in an excellent functional recovery.

\section{Case 4}

A 38 year old Asian man presented with bilateral sciatic pain. Radiographs of the spine showed only minor degenerative changes, and the chest film was normal. His pain continued, and he developed weakness of eversion of the left foot, with sensory impairment on the lateral aspect of the foot. The left ankle jerk was absent, and both plantar responses were flexor. There was slight tenderness in the lower lumbar region. A lumbar myelogram revealed almost complete extradural obstruction of the column of contrast medium at the level of the body of the fifth lumbar vertebra. Tomograms of this region showed a small destructive lesion of the body of the fifth lumbar vertebra. The adjacent intervertebral disc spaces were normal. The CSF contained $0.5 \mathrm{~g}$ protein $/ 1$ and 4 lymphocytes $/ \mathrm{mm}^{3}$. The ESR was $55 \mathrm{~mm} / \mathrm{hr}$. It was considered that the most likely diagnosis was metastatic neoplasm.

At laminectomy a mass, arising from the body of the fifth lumbar vertebra, was found to contain a creamy fluid, from which acid-fast bacilli were subsequently cultured. Biopsy specimens showed granulomatous inflammatory changes, with giant cells. Postoperatively he was treated with antituberculous chemotherapy, and a slow but full recovery ensued.

Comment Vertebral tuberculosis with extradural compression of the cauda equina by tuberculous granuloma was not suspected before operation. The CSF protein was normal, and the minimally raised cell count in the CSF did not arouse suspicion. Although the tuberculous granuloma was opened and partly excised without a preliminary period of treatment with antituberculous drugs, meningitis did not follow the operation. 
INTRADURAL SPINAL TUBERCULOMA

Extradural tuberculomas usually arise as a comlication of tuberculosis of the spine (see cases 3 and 4) although a few cases have been reported in which no bony involvement or extradural component was discovered (Tandon and Pathak, 1973). Intradural spinal tuberculomas, however, are uncommon. Dastur (1972) found only six examples in a series of 266 tuberculomas of the central nervous system. We have observed one patient in whom paraplegia caused by cord comprescion from intradural tuberculoma seems to have developed as a complication of tuberculous meningitis.

\section{Case 5}

This 24 year old Asian man had come to Britain from Tanzania in 1963. In 1970 he developed pulmonary tuberculosis, complicated by tuberculous meningitis. Acid-fast bacilli were isolated from both sputum and CSF. During treatment with streptomycin, isoniazid, and para-amino salicylic acid he suddenly developed moderate flaccid weakness of both legs, with reduced tendon reflexes, but with extensor plantar responses. There was no pain or sensory loss. The CSF protein at this time was $25 \mathrm{~g} / 1$. This weakness recovered during a period of about two weeks without change in management.

Two years later, while taking antituberculous chemotherapy, he presented with a three month history of increasing weakness and numbness of his legs, with urgency and frequency of micturition. For two weeks he had noticed persistent pain in the middle of his back and for a week he had been unable to stand unaided. On examination there was a dense flaccid paraplegia with extensor plantar responses. The tendon reflexes were absent. There was dense sensory impairment to all modalities below a level at T12 dermatome. Radiographs of the spine were normal. The chest radiograph showed pleural thickening, but no evidence of active pulmonary tuberculosis. A lumbar myelogram showed complete obstruction to the flow of contrast medium at the level of the body of the eleventh thoracic vertebra. At laminectomy a dorsally situated subarachnoid tuberculoma was excised. This tuberculoma consisted of numerous small tuberculoid granulomata which extended over several segments of the cord. The cord was slightly indented by this lesion. His legs remained weak and spastic but bladder control and distal sensation returned.

Comment In this patient a zone of intradural granuloma and arachnoiditis extended over several spinal segments. In most previous reports this disorder has presented as a progressive paraplegia without a history of meningitis (Tandon and
Pathak, 1973). In some of these cases localised solid tuberculoma compressed the cord (Arseni and Samitcar, 1960; Jenkins and Hill, 1963; Parsons and Pallis, 1965), but in others a more d:ffuse granulomatous mass surrounded the cord and was associated with localised arachnoiditis (Bucy and Oberhill, 1950). Our case seemed to combine features of both these descriptions.

TUBERCULOUS RADICULOMYELITIS (ARACHNOIDITIS) Tuberculous radiculomyelitis occurs typically as the first manifestation of tuberculosis of the central nervous system (Wadia, 1973b). This occurred in three of our patients.

\section{Case 6}

This 18 year old Asian had entered Britain from his native Bangladesh three weeks before his admission to The London Hospital. For six weeks he had observed weakness in his arms and legs, and for two weeks he had been unable to walk unaided. During this time he had developed difficulty in micturition with occasional incontinence. He also complained of sharp pains in his arms and legs, especially in a sciatic distribution. On examination there was a flaccid quadriparesis, affecting the legs more than the arms. The tendon reflexes were absent in the legs, but the plantar responses were flexor. There was no sensory loss. Radiographs of the chest and spine, and a myelogram were normal. The Mantoux test was negative. The CSF contained $0.47 \mathrm{~g}$ protein/1 and 46 lymphocytes $/ \mathrm{mm}^{3}$ but a second specimen taken a week later contained $2.6 \mathrm{~g}$ protein $/ 1,480$ leucocytes (mainly lymphocytes) $/ \mathrm{mm}^{3}$, and $2.1 \mathrm{mmol}$ glucose /1. The blood glucose was $5.0 \mathrm{mmol} / 1$. During this short period of observation he deteriorated so that he was unable to stand without assistance. Treatment was, therefore, started with rifampicin, ethambutol, isoniazid, and pyridoxine. He gradually improved, and was discharged 10 weeks later walking unaided.

Two months later there was no clinical abnormality, although the CSF contained $0.9 \mathrm{~g}$ protein $/ 1$ and 10 lymphocytes $/ \mathrm{mm}^{3}$. The CSF glucose at this time was $5.0 \mathrm{mmol} / \mathrm{l}$. Acid-fast bacilli were never isolated from CSF or urine. Antituberculous chemotherapy was continued for a further period of six months, and two years later he was well and in fulltime employment.

\section{Case 7}

This patient, an Asian aged 48 years, who had moved to Britain from India several years previously, presented with a five week history of progressive paraplegia, impaired sensation in the legs, 
pains in buttocks and thighs, and impotence. Urinary incontinence developed a few days before admission.

On examination there was a flaccid paraparesis, with sensory impairment to light touch below a vague level at the mid thigh bilaterally. Position sense was impaired in the toes, but painful stimuli were relatively well perceived. The ankle jerks could scarcely be elicited but the knee jerks were brisk and both plantar responses were extensor. The bladder was palpable and required catheterisation. The Mantoux test was positive at $1: 1000$. A radiograph of the chest revealed a calcified hilar gland, but films of the spine and a myelogram were within normal limits. The CSF contained $2 \mathrm{~g}$ protein $/ 1,150$ leucocytes (mainly lymphocytes) $/ \mathrm{mm}^{3}$, and $2.5 \mathrm{mmol}$ glucose $/ 1$. He was treated with rifampicin, ethambutol, and isoniazid, and during the next few weeks strength in his legs slowly improved, and voluntary micturition returned. However, his legs remained weak and he continued to complain of impotence, urinary frequency, and urgency. Acid-fast bacilli were never isolated from the CSF or urine.

\section{Case 8}

This 61 year old Englishman, who had lived all his life in London, was transferred from another hospital for investigation of weakness of both legs. Six months previously he had noted low back pain, radiating into the left leg in a sciatic distribution, and followed a few weeks later by weakness and numbness of the left foot. There had been no previous injury. He gradually became aware of increasing weakness, affecting both legs. On examination there was a severe flaccid paraparesis. The left ankle jerk was absent but the other tendon reflexes in the legs were increased. There was sensory loss in the left L5 dermatome. The spine was normal. Straight leg raising was painless to $70^{\circ}$ in both legs. The chest radiograph was normal. Plain films of the spine revealed spondylotic changes in the cervical and lumbar regions with narrowing of the L5/S1 intervertebral space, but there was no bony erosion at any level. A metrizimide myelogram showed a small filling defect at the left L5/ S1 intervertebral disc, and thickening of all the lower lumbar and sacral nerve roots. The CSF contained more than $2 \mathrm{~g}$ protein $/ 1,163$ leucocytes (mainly lymphocytes) $/ \mathrm{mm}^{3}$, and $2.5 \mathrm{mmol}$ glucose /1. The ESR was $45 \mathrm{~mm} / \mathrm{hr}$.

Carcinomatous meningitis was considered the most likely diagnosis but cytological examination of the CSF and sputum failed to show malignant cells. An exploratory laminectomy was performed. The roots of the cauda equina were thickened and reddened. A biopsy of the meninges revealed a granulomatous inflammatory response, without giant cells or acid-fast bacilli. Cultures of the biopsy specimen, and of the CSF failed to grow acid-fast or other bacilli, or fungi. The Mantoux test was positive at $1: 1000$. A liver biopsy was normal and the Kveim test negative. Serological tests for syphilis were negative.

After the laminectomy he continued to deteriorate, so that he became unable to stand, and had to be catheterised. Sensory impairment developed below a level at about T10 dermatome. Antituberculous chemotherapy consisting of rifampicin, streptomycin, and isoniazid was started but there was no improvement. Four weeks later a right hemiplegia, without visual field defect or aphasia, developed. Steroid therapy was added without effect on the paraparesis, but the hemiplegia gradually improved.

Comment In case 8 the diagnosis remained uncertain. It was assumed that the granulomatous myeloradiculitis was caused by tuberculous infection, since fungal infection, syphilis, carcinoma, and sarcoidosis had been excluded. There was no evidence of systemic vasculitis, of lumbosacral disc protrusion, or of Pott's disease. Despite treatment with antituberculous drugs and steroids there was no improvement.

TUBERCULOSIS OF THE CENTRAL NERVOUS SYSTEM 50 YEARS AGO

We have examined the microfilmed case records of all patients with tuberculosis of the central nervous system admitted to The London Hospital in the years 1920 and 1921 . There were 66 patients. The majority were children (mean age 8.5 years), and nearly all were of English or European origin. Thirty-six were female.

All these patients suffered from tuberculous meningitis and all died; $94 \%$ survived for less than two weeks after admission. In $52(79 \%)$ the illness lasted less than two weeks before diagnosis, but in the remainder there was a nonspecific prodromal illness lasting one to three months, consisting principally of malaise and weight loss. Sixty-two patients presented with the clinical syndrome of uncomplicated meningitis. In four patients there were additional features. One of these developed focal epilepsy, two had hemiplegia, and one paraplegia. The CSF was examined in 51 of the 66 patients. The CSF protein was invariably raised (but it could not be measured accurately at this time), and the cell count ranged from 8 to 381 leucocytes $/ \mathrm{mm}^{3}$ (mean $85 / \mathrm{mm}^{3}$ ). Acid-fast bacilli were found in smears of the CSF sediment in 15 cases $(29 \%)$. 
Postmortem examination was performed in 42 cases. In all of them there was tuberculous meningitis with pulmonary and miliary tuberculosis. There was hydrocephalus in one case. Solitary cerebral tuberculomas were found in six cases; one of these patients had focal epilepsy, and the other had a hemiplegia. The other patient with a hemiplegia had tuberculous arteritis of the middle cerebral artery.

\section{Discussion}

The clinical distinction between the various forms of spinal tuberculosis with paraplegia is difficult to maintain in practice (Tandon and Pathak, 1973). In recognising the similar clinical and pathological features of these patients, Wadia and Dastur (1969) and Wadia (1973b) have suggested that the designation tuberculous radiculomyelitis be used as a generic term to include cases previously categorised as arachnoiditis (Ransome and Monteiro, 1947), intradural spinal tuberculoma (Jenkins and Hill, 1963; Parsons and Pallis, 1965), or granuloma (Bucy and Oberhill, 1950; Arseni and Samitcar, 1960), or as spinal cord complications of tuberculous meningitis (Brooks et al., 1954; Dibble and Cascino, 1956; Bawa and Wahi, 1961; Kocen and Parsons, 1970). These patients' tuberculous paraplegias differ, however, both in origin and course, from those caused by primary tuberculous radiculomyelitis. Wadia (1973b) has taken account of this difference and has suggested the following classification: (1) primary tuberculous spinal radiculomyelitis (cases 6,7, and 8); (2) radiculomyelitis secondary to tuberculous basal meningitis (cases 1 and 5); (3) radiculomyelitis secondary to vertebral tuberculosis (cases 2, 3, and 4).

This concept allows recognition of the almost invariable combination of root and cord signs in patients with lumbar infection, accounting for the local and radicular pain, and the flaccid, areflexic paraparesis with distal sensory impairment and extensor plantar responses. The observation that spinal block often occurs before the development of paraplegia (Wilson, 1940; Brooks et al., 1954) both in patients with classical tuberculous meningitis (cases 1 and 2) and in patients with vertebral tuberculosis (cases 3 and 4 ) is thus easily understood.

Primary tuberculous radiculomyelitis accounted for 20 of the 38 cases of tuberculous radiculomyelitis reported by Wadia and Dastur (1969), but it has not been recognised in this country. Indeed, Elkington $(1936,1951)$ entirely discounted tuberculosis as a cause of arachnoiditis. Our cases 6, 7, and 8 were typical examples of the disorder. In case 8 , however, the tuberculous origin of the radiculomyelitis could not be established, even after meningeal biopsy. Syphilis, pyogenic, or fungal infection, carcinomatous meningitis, and a variety of noninfective agents may cause this syndrome, and a similar localised spinal arachnoiditis may occur rarely after myelography, or after other intrathecal infections, particularly in the presence of other spinal disorders. (Shaw et al., 1978). Nonetheless, Wadia and Dastur (1969) failed to find a cause in 10 of their 70 cases. Primary tuberculous radiculomyelitis probably arises as a localised arachnoiditis from a primary focus in the meninges (Rich, 1951). The CSF changes are thus typical of an indolent meningitis; indeed, the CSF glucose may be normal (Wadia and Dastur, 1969; Wadia, 1973b). Myelography usually reveals multiple, irregular filling defects, cyst formation, or spinal block (Wadia, 1973b; Shaw et al., 1978). Treatment with antituberculous drugs may need to be prolonged, and some patients show little improvement (Wadia, 1973b). However, two (cases 6 and 7) of our three cases of primary tuberculous radiculomyelitis made an excellent functional recovery. Four (cases 1, 3, 4, and 5) of our five patients with radiculomyelitis secondary to vertebral tuberculosis or to tuberculous meningitis also recovered substantially. The role of steroid therapy is uncertain, but in case 1 there seemed to be a rapid improvement when steroids were started.

Arachnoiditis (radiculomyelitis), usually due to tuberculosis (Wadia, 1973b), is a common cause of paraplegia in India (Ramamurthi, 1961; Mani et al., 1969) but similar spinal complications of tuberculosis have, until recently, been unusual in Britain, as shown by the experience at The London Hospital 50 years ago, when tuberculous meningitis was a uniformly fatal disease. However, spinal tuberculomas are recorded in the older English literature (Hunter, 1902; Horsley, 1909), and Wilson (1940) recognised that transverse myelitis could occur both as a presenting feature, and as a complication of tuberculous meningitis. Our current experience of these disorders reflects an interplay of several factors, including differences in host immunity because of improved nutritional status, survival from acute tuberculous infection resulting from effective antituberculous treatment, and the arrival of Asian immigrants who seem peculiarly liable to develop subacute or chronic forms of the disease.

\section{References}

Arseni, C., and Samitcar, D. C. (1960). Intraspinal tuberculous granuloma. Brain, 83, 285-292. 
Bawa, Y. S., and Wahi, P. L. (1961). Spinal tuberculous meningitis. Journal of the Indian Medical Association, 37, 449-452.

Brooks, W. D. W., Fletcher, A. P., and Wilson, R. R. (1954). Spinal cord complications of tuberculous meningitis: a clinical and pathological study. Quarterly Journal of Medicine, NS, 23, 275-290.

Bucy, P. C., and Oberhill, H. R. (1950). Intradural spinal granulomas. Journal of Neurosurgery, 7, 1-12.

Dastur, H. M. (1972). A tuberculoma review with some personal experiences. II. Spinal cord and its coverings. Neurology (India), 20, 127-131.

Dastur, D. K., Lalitha, V. S., and Prabhakar, V. (1968). Pathological analysis of intracranial spaceoccupying lesions in 1000 cases including children. Part 1: age, sex and pattern; and the tuberculomas. Journal of the Neurological Sciences, 6, 575-593.

Dastur, D. K., and Wadia, N. H. (1969). Spinal meningitides with radiculomyelopathy. Part 2: pathology and pathogenesis. Journal of the Neurological Sciences, 8, 261-297.

Dibble, J. B., and Cascino, J. (1956). Tuberculoma of the spinal cord. Journal of the American Medical Association, 162, 461-462.

Elkington, J. St. C. (1936). Meningitis circumscripta spinalis. Brain, 59, 181-203.

Elkington, J. St. C. (1951). Arachnoiditis. In Modern Trends in Neurology, vol. 1. Edited by A. Feiling. Butterworth: London.

Horsley, V. (1909). Chronic spinal meningitis: its differential diagnosis and surgical treatment. British Medical Journal, 1, 513-517.

Hunter, W. K. (1902). A case of tubercular tumour of the spinal cord in a child two years old. Brain, 25, 226-239.

Jenkins, R. B., and Hill, C. (1963). Intradural spinal tuberculoma with genito-urinary symptoms. Archives of Neurology (Chicago), 8, 539-543.

Kocen, R. S., and Parsons, M. (1970). Neurological complications of tuberculosis: some unusual manifestations. Quarterly Journal of Medicine, NS, 39, 17-30.
Mani, K. S., Mani, A. J., and Montgomery, R. D. (1969). A spastic paraplegia syndrome in South India. Journal of the Neurological Sciences, 9, 179199.

McNicol, M. W., Mikhail, J. R., and Sutherland, I. (1971). Tuberculosis in Brent. Postgraduate Medical Journal, 47, 691-693.

Parsons, M., and Pallis, C. A. (1965). Intradural spinal tuberculomas. Neurology (Minneapolis), 15, 10181022.

Ramamurthi, B. (1961). Spinal arachnoiditis. Indian Journal of Medical Sciences, 15, 776-781.

Ransome, G. A., and Monteiro, E. S. (1947). A rare form of tuberculous meningitis. British Medical Journal, 1, 413-414.

Rich, A. R. (1951). The Pathogenesis of Tuberculosis. Second edition. Oxford University Press: London.

Scadding, J. (1967). Sarcoidosis. Eyre and Spottiswoode: London.

Shaw, M. D. M., Russell, J. A., and Grossart, K. W. (1978). The changing pattern of spinal arachnoiditis. Journal of Neurology, Neurosurgery, and Psychiatry, 41, 97-107.

Tandon, P. N., and Pathak, S. N. (1973). Tuberculosis of the central nervous system. In Tropical Neurology, pp. 37-62. Edited by J. D. Spillane. Oxford University Press: London.

Wadia, N. H. (1973a). An introduction to neurology in India. In Tropical Neurology, pp. 25-36. Edited by J. D. Spillane. Oxford University Press: London.

Wadia, N. H. (1973b). Radiculomyelopathy associatedo with spinal meningitides (arachnoiditis) with special reference to the spinal tuberculous variety. In Tropical Neurology, pp. 63-72. Edited by J. D. Spillane. Oxford University Press: London.

Wadia, N. H., and Dastur, D. K. (1969). Spinal meningitides with radiculo-myelopathy. Part 1: clinical and radiological features. Journal of the Neurological Sciences, 8, 239-260.

Wilson, S. A. K. (1940). Neurology. Edward Arnold: London. 